\title{
Do Thyroid Hormones have any Effects on the Development of Retinopathy of Prematurity?
}

\author{
Yeşim Coşkun ${ }^{1}$, ๑ Özge Yabaş Kızıloğlu², ๑ Tevfik Bayram ${ }^{3}$, $\odot$ Eylem Demirci ${ }^{4}$, $\odot$ Ijpek Akman ${ }^{1}$ \\ ${ }^{1}$ Department of Pediatrics, Bahcesehir University Faculty of Medicine, Istanbul, Turkey \\ ${ }^{2}$ Department of Ophthalmology, Bahcesehir University Faculty of Medicine, Istanbul, Turkey \\ ${ }^{3}$ Department of Public Health, Marmara University Faculty of Medicine, Istanbul, Turkey \\ ${ }^{4}$ Department of Pediatrics, Bahcesehir University Medical Park Goztepe Hospital, Istanbul, Turkey
}

\begin{abstract}
Introduction: Retinopathy of prematurity (ROP) is a disease characterized by abnormal vascularization of the retina in preterm infants. The present study aims to investigate the relationship between ROP and serum thyroid hormones based on the known effects of thyroid hormones on angiogenesis.

Methods: This retrospective study included infants born <34 weeks of gestation between January 2011 and January 2019 who were hospitalized from birth to at least the first month of life in the Neonatal Intensive Care Unit and who had regular ROP examinations. The patients were divided into two groups as having ROP and no ROP. Patients diagnosed as ROP were divided into two subgroups as follows: treated ROP and untreated ROP. Variables, such as gender, gestational age (GA), birth weight (BW), bronchopulmonary dysplasia (BPD), duration of mechanical ventilation, duration of oxygen therapy, necrotizing enterocolitis and intraventricular hemorrhage (IVH), together with serum-free T4 and TSH levels on postnatal $7^{\text {th }}$ (sT4-1 and TSH-1) and $28^{\text {th }}$ days (ST4-2 and TSH-2), were examined.

Results: One hundred ninety-three infants without ROP (Group 1) and 152 infants with ROP (Group 2) enrolled in this study. Among 152 patients with ROP, 114 infants did not receive ROP treatment (Group 2a), and 38 infants received ROP treatment (Group 2b). In Group 2, free T4-1 and TSH-2 were significantly lower than in Group 1 ( $p<0.001)$. The sT4-1 and sT4-2 levels of Group 2b were significantly lower than those of Group 2a ( $p=0.042$ and $p=0.015$, respectively). In the logistic regression analysis of risk factors, thyroid hormone levels were not found to be independently effective on the development of the ROP. Discussion and Conclusion: In this study, the findings suggest that thyroid hormones had no effect on ROP development. Larger and prospective studies may shed light on the effects of thyroid hormones on ROP development.

Keywords: Prematurity; retinopathy of prematurity; thyroid hormones
\end{abstract}

$\mathrm{R}^{\mathrm{e}}$ etinopathy of prematurity (ROP) is a developmental disorder of retinal vessels of premature babies and its pathophysiology is unknown. ROP is one of the most important causes of visual impairment and blindness in children. Recent advancements in neonatal care have increased the chances of premature infants' survival. Correspondingly, the frequency of ROP has also increased in this group of infants. Reportedly, ROP has been a problem for babies born before 28 gestational weeks (GW) in de- veloped countries, whereas ROP increases up to $34 \mathrm{GW}$ in developing countries ${ }^{[1]}$. A multicenter study conducted in our country in 2014 revealed that the incidence of ROP in low birth-weight preterm infants was $42 \%$, and the incidence of advanced-stage ROP was $8.2 \%$. In the same study, the incidence of ROP was found to be $13.3 \%$ and the incidence of advanced-stage ROP was $0.4 \%$ in babies over 32 GW. Early diagnosis and treatment of ROP reduce the risk of retinal detachment and blindness ${ }^{[2]}$.

Correspondence (İletişim): Yeşim Coşkun, M.D. Bahcesehir Universitesi Tip Fakultesi, Cocuk Sagligi ve Hastaliklari Anabilim Dali, Istanbul, Turkey Phone (Telefon): +90 5322556479 E-mail (E-posta): coskunyesim@yahoo.com 
Although there are various views on the pathogenesis of premature retinopathy, the biphasic theory proposed by Aston and Smith is the most widely acknowledged hypothesis $^{[3,4]}$. According to this theory, ROP occurs in two phases. Phase 1 is the phase in which vaso-obliteration occurs. In this phase, it is thought that significant angiogenic factors, such as the vascular endothelial factor (VEGF) and the insulin-like growth factor-1 (IGF-1), have an effect on angiogenesis. Phase 2 is the phase of neovascularization. In this phase, an increase in the secretion of angiogenic factors is observed due to hypoxia. It is believed that the main responsible period for ROP development is Phase 2 , where neovascularization occurs.

The thyroid hormone is necessary for normal development of the human brain during the intrauterine period and the first two years of life ${ }^{[5]}$. As the gestational week increases, the amount of serum-free thyroxine (fT4) and thyrotropin (TSH) synthesized by the fetus increases. Thyroid functions in preterm infants are affected by the immaturation of the hypothalamo-pituitary-thyroid axis, diseases and drugs used. In babies that are born mature, fT4 and TSH levels increase after birth then begin to decrease rapidly within 24 hours and continue to slow down after 48 hours. This fluctuation in thyroid functions is less common in low GW neonates ${ }^{[6-9]}$.

Thyroid hormones have also been implicated to have a role in angiogenesis ${ }^{[10-12]}$. In this study, we aimed to investigate the effects of thyroid hormones on ROP development based on this information.

\section{Materials and Methods}

This study was retrospectively carried out with $34 \mathrm{GW}$ patients who were hospitalized between January 2011 and January 2019 at Bahçeşehir University, Faculty of Medicine Göztepe Medical Park Hospital Neonatal Intensive Care Unit (NICU). The research protocol was approved by the Ethics Committee of Bahçesehir University Faculty of Medicine (No: 2019-05/10).

\section{Research Groups}

This research included patients who were born prematurely before the $34^{\text {th }} \mathrm{GW}$ and who were hospitalized in the NICU for more than one month from birth and underwent ROP examinations. The patients were divided into two groups as per their diagnosis of ROP. Patients who were diagnosed with ROP were also divided into two following subcategories: patients who received treatment and patients who did not. Patients who died during the course of this research, patients who underwent exchange transfusion or patients with missing medical records were excluded from this study. The first ophthalmologic examination of the patients was performed by an experienced ophthalmologist (O.Y.K.) for infants younger than $27 \mathrm{GW}$ in the $30^{\text {th }}$ or $31^{\text {st }}$ postmenstrual week, for infants born in $27^{\text {th }}$ or the following weeks in postnatal week 4, in accordance with the recommendations of the American Academy of Pediatrics and Turkish Society of Neonatology ${ }^{[1]}$. One hour before the examination, $0.5 \%$ tropicamide and $2.5 \%$ phenylephrine were instilled three times with 15-minute intervals and pupillary dilatation was ensured. For topical anesthesia, $0.5 \%$ proparacaine hydrochloride drop was instilled just before the examination. An anterior segment followed by a fundus examination was made with a binocular indirect ophthalmoscope. The classification of the disease was staged considering the degree of vascular proliferation according to ICROP[14] (International Classification of Retinopathy of Prematurity) criteria: (1) Stage 1: Demarcation line separating the vascular and avascular retina from each other, (2) Stage 2: Ridge: pocked composition, (3) Stage 3: Extraretinal fibrovascular proliferation on the ridge, (4) Stage 4A: Partial retinal detachment (no macular involvement), (5) Stage 4B: Partial retinal detachment (with macular involvement), (6) Stage 5: Total retina decollement. Stage 3 and above, or "plus" disease (increased arterioles folding in the posterior pole of the retina and venous dilatation), were considered severe ROP. Cases with stage I or stage 2 ROP and plus disease in Zone I, stage 3 ROP in Zone I, stage 2 or stage 3 ROP in Zone II and plus disease were considered as treatment indications for ROP ${ }^{[15]}$. Treatment was administered by intravitreal injection of the antiVEGF agent or laser photocoagulation.

\section{Factors Examined in this Research}

Along with factors, such as gender, GW, birth weight (BW), bronchopulmonary dysplasia (BPD), duration of mechanical ventilation (MV), duration of oxygen therapy, necrotizing enterocolitis (NEC) and intraventricular hemorrhage (IVH), fT4 and TSH levels on postnatal $7^{\text {th }}$ and $28^{\text {th }}$ days were also examined.

GW was calculated according to the mother's last menstrual date or Ballard score. Patients with oxygen therapy or mechanical ventilation support longer than 28 days were diagnosed as BPD. The diagnosis of IVH was made by transfontanelle ultrasonography and was staged with the Papile classification. The diagnosis of NEC was made according to the modified Bell criteria in lieu of the clinical and radiological findings. Transient decrease in fT4 levels without increased TSH levels of the premature was diagnosed as transient hypothyroxinemia, low fT4 level according to ges- 
tational age, and patients with high TSH levels were diagnosed as congenital hypothyroidism.

\section{Laboratory Methods}

On the patient's postnatal $7^{\text {th }}$ day and $28^{\text {th }}$ day, a $2 \mathrm{ml}$ venous blood sample was taken on an empty stomach into the sterile dry tube in the morning and sent to the laboratory. Blood samples were centrifuged within one hour to separate the serum. On the same day, serum TSH and fT4 levels were studied with the chemiluminescence microparticle immunoassay (CMIA) method. Samples were studied with the Abbott ARCHITECT ci8200 system. The ones researched on postnatal day 7 were called sT4-1 and TSH-1, and the ones on postnatal day 28 were called sT4-2 and TSH-2.

\section{Statistical Analysis}

The data were analyzed using the SPSS program. Comparisons were mainly made between three groups: non-ROP patients, patients who are ROP but are not receiving treatment, and patients who are ROP but are receiving treatment. Kruskal Wallis test was used for continuing factors in triple comparisons. In cases where there was a statistically significant difference in triple comparisons $(p<0.05)$, dual comparisons were made with post-hoc analyzes. MannWhitney $\mathrm{U}$ test was used for post-hoc analysis, and significant P-value was accepted as 0.017 (0.05/3) with Bonferroni correction. Chi-square test was used for the analysis of categorical variables. The variables that were deemed significant in univariate analyses were also evaluated by multivariate Logistic Regression models.

\section{Results}

One hundred ninety-three premature infants without ROP disease (Group 1) and 152 premature infants with ROP disease (Group 2) were included in this study. Table 1 shows the comparison of demographic characteristics and variables

Table 1. Comparison of the demographic characteristics and variable factors in patients with and without ROP

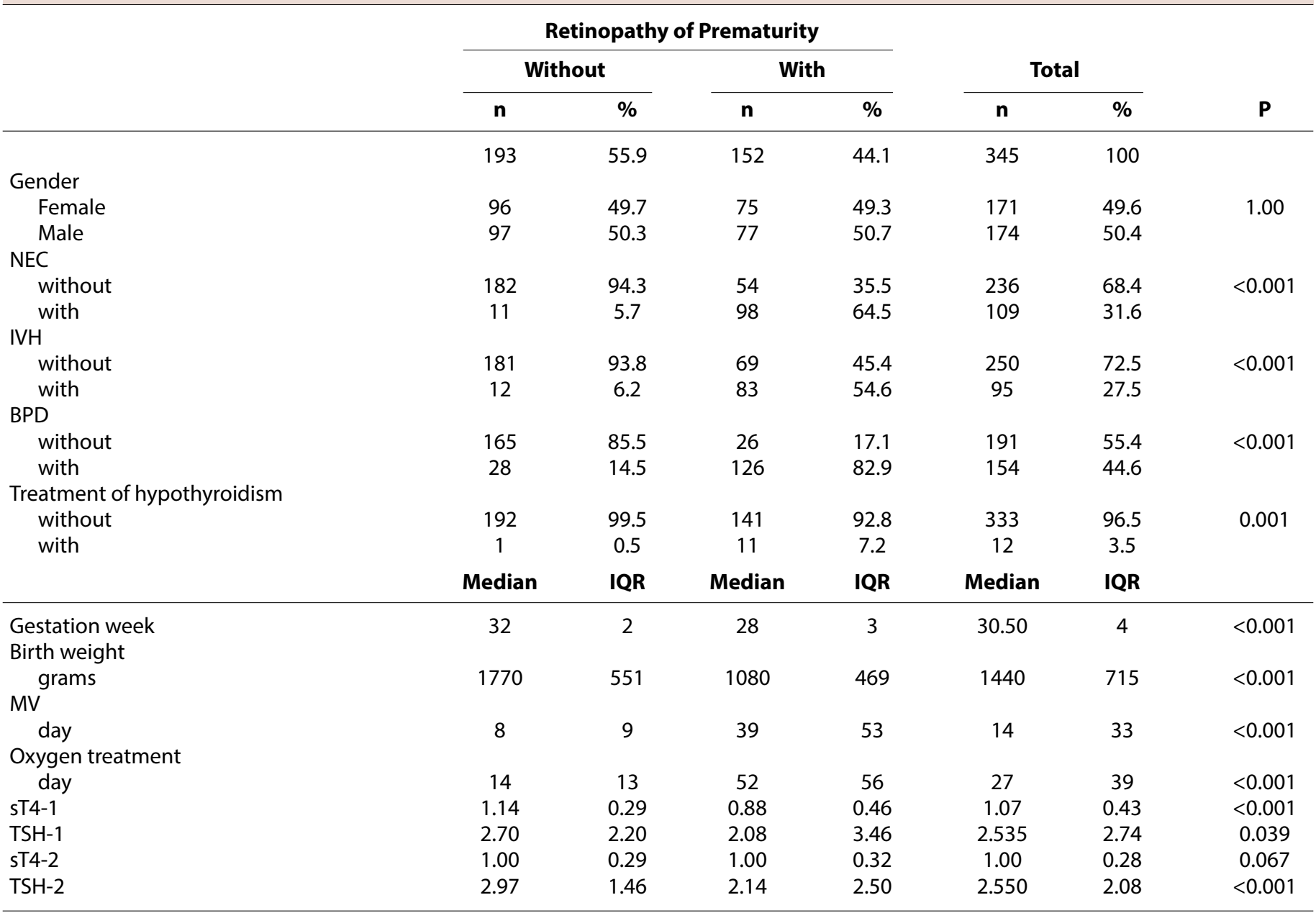

NEC: Necrotizing enterocolitis; IVH: Intraventricular hemorrhage; BPD: Bronchopulmonary dysplasia; MV: Mechanical ventilation; sT4-1: free T4 value at day 7; sT4-2: free T4 value on the $28^{\text {th }}$ day; TSH-1: $7^{\text {th }}$ day TSH value; TSH-2: TSH value on the $28^{\text {th }}$ day. 
of Group 1 and Group 2 patients. Among 152 patients with ROP, 114 infants did not receive treatment for ROP (Group 2a) and 38 infants received treatment for ROP (Group 2b). The comparison of these subgroups concerning characteristics and varying factors is summarized in Table 2.

In Group 2, GW, BW, duration of MV and duration of oxygen treatment were statistically significant on ROP development compared to Group 1 ( $p<0.001$ for each variable). When these two groups were examined, the presence of NEC, IVH and BPD were found to be statistically significant variables in the development of ROP $(p<0.001)$. In Group 2, sT4-1 and TSH-2 were found to be lower than in Group 1 and were statistically significant $(p<0.001)$. There was no statistically significant difference between the two groups concerning TSH-1 and sT4-2. In Group 2b, BW, $\mathrm{MV}$ and having had oxygen therapy longer than 10 days was statistically significant ( $p=0.043, p=0.003, p=0.002)$. In group $2 b$, NEC was found to be a risk factor ( $p=0.049)$.
IVH and BPD were not statistically significant. The sT4-1 and sT4-2 levels of Group 2b were lower than those of Group $2 a$ and were statistically significant. $(p=0.042$ and $p=0.015$, respectively) There was no significant difference between Group 2a and Group 2b concerning TSH-1 and TSH-2 levels.

It was concluded that this situation did not make a statistically significant difference in the development of ROP in babies diagnosed with hypothyroidism $(p<0.001)$. There was no statistically significant difference in the diagnosis and treatment of hypothyroidism between treated and untreated groups $(p=0.468)$.

When logistic regression analysis was used to determine the risk factors for ROP development, thyroid hormone levels were not concluded to be independent risk factors, and GW, BW, IVH and BPD were concluded to be statistically significant independent risk factors $(p<0.001, p<0.001$, $p=0.015, p=0.012$, respectively) (Table 3 ).

Table 2. Comparison of the demographic characteristics and variable factors of ROP patients with and without treatment

\begin{tabular}{|c|c|c|c|c|c|c|c|}
\hline & \multicolumn{4}{|c|}{ Retinopathy of Prematurity } & & & \multirow[b]{2}{*}{$\mathbf{P}$} \\
\hline & $\mathbf{n}$ & $\%$ & $\mathbf{n}$ & $\%$ & $\mathbf{n}$ & $\%$ & \\
\hline \multicolumn{8}{|l|}{ Gender } \\
\hline Female & 56 & 49.1 & 19 & 50.0 & 75 & 49.3 & 1.00 \\
\hline Male & 58 & 50.9 & 19 & 50.0 & 77 & 50.7 & \\
\hline with & 68 & 59.6 & 30 & 78.9 & 98 & 64.5 & \\
\hline \multicolumn{8}{|l|}{ IVH } \\
\hline without & 56 & 49.1 & 13 & 34.2 & 69 & 45.4 & 0.134 \\
\hline with & 58 & 50.9 & 25 & 65.8 & 83 & 54.6 & \\
\hline \multicolumn{8}{|l|}{ BPD } \\
\hline without & 20 & 17.5 & 6 & 15.8 & 26 & 17.1 & 1.00 \\
\hline with & Median & IQR & Median & IQR & Median & IQR & \\
\hline Gestation week & 28 & $27-30$ & 28 & $26-30$ & 28 & $27-30$ & 0.093 \\
\hline \multicolumn{8}{|l|}{ Birth weight } \\
\hline grams & 1100 & $900-1326$ & 910 & $780-1255$ & 1080 & $845-1315$ & 0.043 \\
\hline \multicolumn{8}{|l|}{ MV } \\
\hline day & 35 & $18-66$ & 69 & $29-115$ & 39 & $20-72$ & 0.003 \\
\hline \multicolumn{8}{|c|}{ Oxygen treatment } \\
\hline day & 46 & $33-80$ & 81 & $45-124$ & 52 & $33-88$ & 0.002 \\
\hline sT4-1 & 0.92 & $0.67-1.17$ & 0.75 & $0.63-0.98$ & .88 & $0.66-1.13$ & 0.042 \\
\hline TSH-1 & 2.09 & $0.94-4.52$ & 1.97 & $0.47-4.05$ & 2.08 & $0.71-4.20$ & 0.403 \\
\hline sT4-2 & 1.02 & $0.84-1.14$ & 0.92 & $0.74-1.03$ & 1.00 & $0.80-1.11$ & 0.015 \\
\hline
\end{tabular}

NEC: Necrotizing enterocolitis; IVH: Intraventricular hemorrhage; BPD: Bronchopulmonary dysplasia; MV: Mechanical ventilation; sT4-1: free T4 value at day 7; sT4-2: free T4 value on the $28^{\text {th }}$ day; TSH-1: $7^{\text {th }}$ day TSH value; TSH-2: TSH value on the $28^{\text {th }}$ day. 
Table 3. Logistic regression analysis of the ROP risk factors

\begin{tabular}{lccc}
\hline & Odds Ratio & 95\% Confidence Interval & P \\
\hline Gestation week & 0.523 & $0.393-0.694$ & $<0.001$ \\
Birth weight & 0.997 & $0.996-0.999$ & $<0.001$ \\
IVH & 3.444 & $1.271-9.335$ & 0.015 \\
BPD & 2.757 & $1.246-6.099$ & 0.012 \\
\hline
\end{tabular}

Model P-value $<0.001 ;$ P-value of Hosmer and Lemeshow test: 0.083; Nagelkerke R2: 0.753; Variables included in the model: gestational week, birth weight, duration of oxygen therapy, necrotizing enterocolitis, intraventricular hemorrhage (IVH), bronchopulmonary dysplasia (BPD), sT4 value of the 1st week.

\section{Discussion}

Normal retinal vascularization starts to take place in the mother's womb at the $16^{\text {th }} \mathrm{GW}$ in two phases: The early phase is called vasculogenesis and the late phase is defined as angiogenesis. Maturation is completed when the blood vessels reach nasal ora serrata at $36 \mathrm{GW}$ and temporal ora serrata at $40 \mathrm{GW}$. The vasculogenesis phase is independent of hypoxia and is the phase in which primitive vascular structure occurs. In angiogenesis, capillary network formation is seen. Retinal photoreceptor activation and neural tissue development give rise to physiological hypoxia and allow the release of angiogenic factors, such as VEGF and IGF-1 at the physiological level[ ${ }^{[16,17]}$.

Since the neurovascular development of the retina at preterm birth is immature, premature infants are susceptible to ROP development. Both oxygen-dependent and oxygen-independent factors play a role in the development of ROP in premature babies. Factors like non-oxygen-related IGF-1 also interact with VEGF in the development of ROP. Oxygen saturation with premature birth and increased partial oxygen pressure relative to the intrauterine environment create relative hyperoxia for the premature infant. The first phase developing around the $30^{\text {th }}$ and $32^{\text {nd }}$ weeks of the two phases proposed in the pathogenesis of ROP is characterized by this early hyperoxic condition. Blood flow autoregulation is suppressed by the effects of toxic oxygen and angiogenic factors, such as VEGF, are reduced, and vaso-obliteration occurs, which leads to hypoxia in the tissue and thus initiates phase 2 . Pathological hypoxia that occurs in the second phase leads to increased release of angiogenic factors, such as VEGF and the development of abnormal vessels ${ }^{[3,4,17]}$.

The effects of thyroid hormones on blood vessels have been known for many years and have been shown to reduce vascular resistance, have a proangiogenic effect and play a role in angiogenesis. Thyroid hormones affect the expression of VEGF-A and FGF-2 and are effective in angiogenesis. Blood VEGF and FGF-2 levels in postnatal thyroid hormone deficiency were determined low in animal experiments ${ }^{[10-12]}$. The discussion above suggests that thyroid hormones may have an effect on ROP development. In our research, to investigate the effects of thyroid hormones on ROP development, we firstly compared the fT4 and TSH levels that we looked at on the $7^{\text {th }}$ and $28^{\text {th }}$ days of preterm babies born less than $34 \mathrm{GW}$ between groups with and without ROP. To understand its effect on ROP more clearly, we compared the groups that were diagnosed with ROP that were or were not being treated for ROP.

Thyroid functions in the postnatal period are affected by several factors, such as GW, postnatal age, BW, mode of delivery and gender. In a study by Aktaş et al., ${ }^{[8]}$ a positive correlation was found between $\mathrm{GW}$ and $\mathrm{T} 4$ values. It is thought that the interruption of the development of the hypothalamus-pituitary-thyroid axis in fetal life with preterm birth led to thyroid dysfunction. Also, comorbidities such seen in premature cases as sepsis and RDS might affect thyroid hormone synthesis ${ }^{[18,19]}$. In Korkmaz et al.'s study, the findings showed that TSH values of healthy preterm infants and preterm infants with health problems, such as RDS, sepsis and ROP decreased gradually in samples taken on days 1 , 7 and 14, and TSH values were significantly lower in infants with health problems. ${ }^{[20]}$ Chung et al. ${ }^{[21]}$ report that free T4 levels were significantly lower in babies born less than 28 GW and RDS and BPD were more common in these babies than in the control group.

The pathogenesis of diabetic retinopathy has some similarities with ROP pathogenesis. In both cases, vascular damage in the retina, ischemia and VEGF-related neovascularization are involved. Some studies showed that subclinical hypothyroidism increased the risk of diabetic retinopathy in patients with Type 2 diabetes mellitus ${ }^{[22,23]}$. Yang et al. ${ }^{[23]}$ showed that subclinical hypothyroidism was associated with visually threatening diabetic retinopathy. These findings suggest that thyroid hormone values may be related to ROP development.

Our research concluded that TSH measurements from the $7^{\text {th }}$ day and from the $28^{\text {th }}$ day for the group with ROP were significantly lower than the measurements without ROP. For the ROP group, on the $7^{\text {th }}$ and $28^{\text {th }}$ day, the fT4 levels were significantly lower in the treated group than in the non-treated ROP group. However, thyroid hormone levels were not determined to be independent risk factors when analyzed with other risk factors in the development of ROP. 
Korkmaz et al. ${ }^{[24]}$ concluded that there was no significant difference in TSH and fT4 values between treated ROP cases and other infants undergoing ROP screening and pointed out significant differences between the groups in terms of BPD, RDS, IVH and MV duration. In our study, BW, IVH and BPD were also determined to be risk factors through multiple analyzes. These results suggest that low thyroid hormone levels seen in prematurity are related to immaturity and associated morbidity factors, and not directly related to ROP development.

There are some factors limiting this study. Given that this is a single-center study and is designed retrospectively, these aspects limit the results of this study.

\section{Conclusion}

In conclusion, this research concluded that thyroid hormones do not have any effect on the development of ROP. Larger and prospectively designed studies are needed to reveal whether thyroid hormones with known effects on angiogenesis are a risk factor for the development of the ROP.

Ethics Committee Approval: The Ethics Committee of Bahcesehir University School of Medicine provided the ethics committee approval for this study (06.03.2019-2019-05/10).

Peer-review: Externally peer-reviewed.

Authorship Contributions: Concept: Y.C., I.A., O.Y.K.; Design: Y.C., I.A., O.Y.K.,E.D.; Data Collection or Processing:Y.C., E.D., T.B.; Analysis or Interpretation:T.B., I.A.; Literature Search:Y.C., O.Y.K., I.A., T.B., E.D.; Writing: Y.C, I.A., O.Y.K., E.D., T.B.

Conflict of Interest: None declared.

Financial Disclosure: The authors declared that this study received no financial support.

\section{References}

1. Kim SJ, Port AD, Swan R, Campbell JP, Chan RVP, Chiang MF. Retinopathy of prematurity: a review of risk factors and their clinical significance. Surv Ophthalmol 2018;63:618-37.

2. Bas AY, Koc E, Dilmen U; ROP Neonatal Study Group. Incidence and severity of retinopathy of prematurity in Turkey. Br J Ophthalmol 2015;99:1311-4. [CrossRef]

3. ASHTON N, WARD B, SERPELL G. Effect of oxygen on developing retinal vessels with particular reference to the problem of retrolental fibroplasia. Br J Ophthalmol 1954;38:397-432.

4. Smith LE, Wesolowski E, McLellan A, Kostyk SK, D'Amato R, Sullivan $\mathrm{R}$, et al. Oxygen-induced retinopathy in the mouse. Invest Ophthalmol Vis Sci 1994;35:101-11.

5. Morreale de Escobar G, Obregon MJ, Escobar del Rey F. Role of thyroid hormone during early brain development. Eur J Endocrinol 2004;151:U25-37. [CrossRef]

6. Fi Fisher DA, Dussault JH, Sack J, Chopra IJ. Ontogenesis of hypothalamic-pituitary-thyroid function and metabolism in man, sheep, and rat. Recent Prog Horm Res 1976;33:59-116.

7. Fisher DA, Odell WD. Acute release of thyrotropin in the newborn. J Clin Invest 1969;48:1670-7. [CrossRef]

8. Aktas ON, Gursoy T, Soysal E, Esencan E, Ercin S. Thyroid hormone levels in late preterm, early term and term infants: a study with healthy neonates revealing reference values and factors affecting thyroid hormones. J Pediatr Endocrinol Metab 2017;30:1191-6. [CrossRef]

9. van Wassenaer AG, Kok JH, Dekker FW, de Vijlder JJ. Thyroid function in very preterm infants: influences of gestational age and disease. Pediatr Res 1997;42:604-9. [CrossRef]

10. Carmona-Cortés J, Rodríguez-Gómez I, Wangensteen R, Banegas I, García-Lora ÁM, Quesada A, et al. Effect of thyroid hormone-nitric oxide interaction on tumor growth, angiogenesis, and aminopeptidase activity in mice. Tumour Biol 2014;35:5519-26. [CrossRef]

11. Liu X, Zheng N, Shi YN, Yuan J, Li L. Thyroid hormone induced angiogenesis through the integrin av $\beta 3$ /protein kinase $D /$ histone deacetylase 5 signaling pathway. Mol Endocrinol 2014;52:245-54. [CrossRef]

12. Zhang L, Cooper-Kuhn CM, Nannmark U, Blomgren K, Kuhn HG. Stimulatory effects of thyroid hormone on brain angiogenesis in vivo and in vitro. J Cereb Blood Flow Metab 2010;30:323-35. [CrossRef]

13. Fierson WM; American Academy of Pediatrics Section on Ophthalmology; American Academy of Ophthalmology; American Association for Pediatric Ophthalmology and Strabismus; American Association of Certified Orthoptists. Screening examination of premature infants for retinopathy of prematurity. Pediatrics 2018;142. pii: e20183061. [CrossRef]

14. International Committee for the Classification of Retinopathy of Prematurity. The International Classification of Retinopathy of Prematurity revisited. Arch Ophthalmol 2005;123:991-9.

15. Early Treatment For Retinopathy Of Prematurity Cooperative Group. Revised indications for the treatment of retinopathy of prematurity: results of the early treatment for retinopathy of prematurity randomized trial. Arch Ophthalmol 2003;121:1684-94. [CrossRef]

16. Hughes $\mathrm{S}$, Yang $H$, Chan-Ling T. Vascularization of the human fetal retina: roles of vasculogenesis and angiogenesis. Invest Ophthalmol Vis Sci 2000;41:1217-28.

17. Akkoyun I. Pathophysiology of Retinopathy of Prematurity. Turk J Ophthalmol 2012;42:63-7

18. Cavarzere P, Camilot M, Popa FI, Lauriola S, Teofoli F, Gaudino $\mathrm{R}$, et al. Congenital hypothyroidism with delayed TSH elevation in low-birth-weight infants: incidence, diagnosis and management. Eur J Endocrinol 2016;175:395-402. [CrossRef]

19. Dutta S, Singh S, Bhattacharya A, Venkataseshan S, Kumar P. Relation of thyroid hormone levels with fluid-resistant shock among preterm septicemic neonates. Indian Pediatr 2017;54:121-4. [CrossRef]

20. Korkmaz G, Özçetin M, Çağ Y, Yükselmiş U, Öngel V, Işık O. Thyroid function in healthy and unhealthy preterm newborns. $A$ 
Afr Health Sci 2018;18:378-83. [CrossRef]

21. Chung HR, Shin CH, Yang SW, Choi CW, Kim Bl, Kim EK, et al. High incidence of thyroid dysfunction in preterm infants. J Korean Med Sci 2009;24:627-31. [CrossRef]

22. Kim BY, Kim CH, Jung CH, Mok JO, Suh KI, Kang SK. Association between subclinical hypothyroidism and severe diabetic retinopathy in Korean patients with type 2 diabetes. Endocr J
2011;58:1065-70. [CrossRef]

23. Yang JK, Liu W, Shi J, Li YB. An association between subclinical hypothyroidism and sight-threatening diabetic retinopathy in type 2 diabetic patients. Diabetes Care 2010;33:1018-20.

24. Korkmaz L, Baştuğ O, Daar G, Korkut S, Özdemir A, Adnan Öztürk $M$, et al. The effects of thyroid function on retinopathy of prematurity. J Neonatal Perinatal Med 2016;9:349-56. 